\author{
Marek Rosiak
}

\title{
SOME ONTOLOGICAL PROBLEMS CONCERNING PREDICATION
}

\begin{abstract}
The Aristotelian double characterization of a primary substance exploits the difference between the part-whole relation and the non-linguistic relation of predication. A problem arises whether and how the second relation could be reduced to something else. Such a reduction is explicitly declared or at least implicitly assumed in all version of conceptualism and nominalism. The moderate realism is often interpreted as a reductionism of this kind but such interpretations do not seem corect. Only the so called resemblance theory can be regarded as a successful attempt at the reduction in question. However, it seems that the predication per se cannot be disposed of even in this way. If so, then the nature of the relation of predication still waits for a precise analysia and description. This would provide an insight into the essence of a universal being
\end{abstract}


Aristotle charcterizes a PRIMARY (individual) SUBSTANCE (PS) as something which is neither said of nor is in anything (Cat. 2 a 11 nn.: "Substantia [prima] neque de subiecto predicatur neque in subiecto est.") This classical characteristics is, alas, neither definitive nor univocal. "Is said of" ( $\lambda \varepsilon \dot{\gamma \varepsilon \tau \alpha \iota)}$ was submitted to various interpretations and eventually gave rise to me-

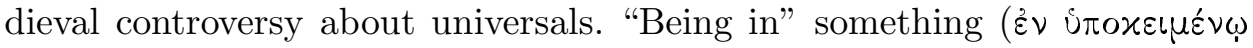
$\tau \iota v i$ Eiv $\alpha$ ) could perhaps be understood as being a component of a thing, i.e. the part-whole relation or its analogue. It seems that Aristotle had in mind two independent conditions imposed on arguments of these relations: the first to safeguard particularity of a prime substance (as opposed to generality of a predicate) and the second to protect its independence. Aristotle's own examples of a particular man or horse seem to support this interpretation. So the starting point for further investigations can be presented as two dichotomies crossing each other:

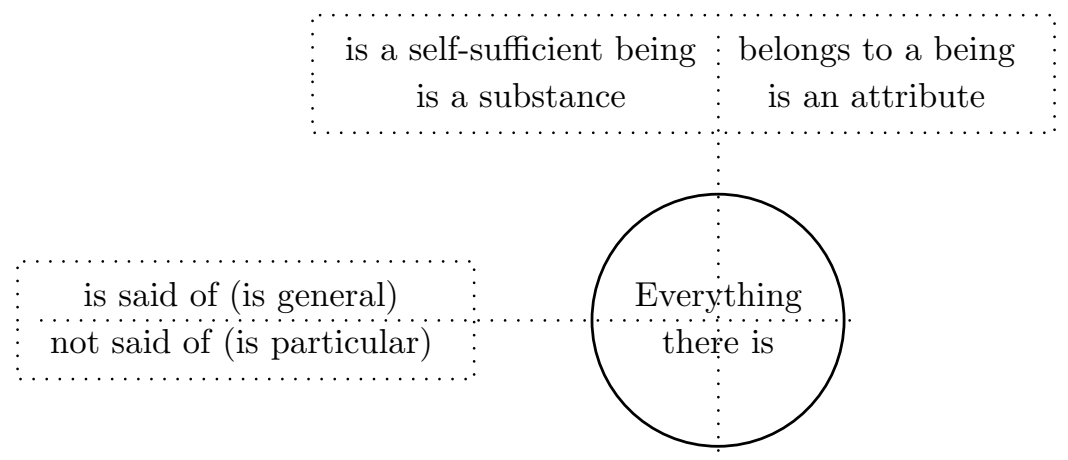

Diag. 1

The second condition introduces the distinction between independent existence and the so called INEXISTENCE, i.e. existence of something which can exist only in a broader context. Such is a PARTICULAR ATTRIBUte (PA) or part of a particular thing, e.g. Socrates' big belly which cannot continue to exist in separation from its owner. ${ }^{1}$ Also a General atTribute (GA) like

\footnotetext{
1 There is undoubtedly a sharp difference between the particular shape of my desk and its top. The first is called an attribute of the desk, the other - its part. An attribute cannot effectively be separated from the subject while a part can (but not as a part of
} 
corpulent cannot exist independently. If we cancel the first condition of being a substance, we get a predicate (not as an expression but as the reference of an expression). An ontological equivalent of its predicative character is generality: whether of a substance (GS, e.g. man) or of an attribute (GA, e.g. corpulent). This generality does not imply actual plurality of the subjects of predication: it only means that what is predicated of one thing can, in principle, be predicated of certain other things. ${ }^{2}$

It is clear that the relation of predication holds between GS and PS (Socrates is a man) and the part-whole relation holds between PA (as a part or an attribute) and PS (Socrates has (his inimitable) big belly and corpulent shape). Moreover, it seems clear that also GA is in relation of predication to PS (Socrates is corpulent). But what is the role of the relevant inexistent constituents (the big belly or individual corpulence) of Socrates here? Without it Socrates would not have been corpulent, yet corpulent is not predicated of the belly but of the integral subject. We can say that corpulent is said of Socrates as a whole but with respect to only one of his parts or aspects. This "partial" basis of this kind of predication is reflected in the grammatical form: an adjective (instead of substantive) used in this situation indicates that not the whole subject but only its some property or group of properties are taken into account. Of course, referring to an attribute, we cannot ignore its inexistence and thus we refer indirectly to the subject which supports it. But the subject is referred to only as the mere supporter of the relevant attribute. When GS is predicated of PS, it is said with respect to the whole subject: manhood is neither a part nor an aspect of Socrates. It does not belong to Socrates, although Aristotle says that it is in Socrates. ${ }^{3}$ But is it not the case that when we say Socrates is a man, we take into account only his essential attributes and ignore contingent properties? The answer is that they are referred to indirectly, since it is impossible for a subject to be destituted of all contingent properties (e.g. for a man

course). But they both share the character of a particular component of some bigger unity. Because of that common character both were called parts in Brentanist tradition. This must have been precisely the same observation behind Aristotelian description of something which is in the subject but is not said of it.

${ }^{2}$ The relation holding between particulars and relevant universals is the relation of exemplification, participation or falling under. These terms could be used instead of predication to avoid linguistic connotations.

${ }^{3}$ This statement, characteristic for moderate realism of Aristotle raises a question of the nature of the relation between universals and particulars. We shall deal with it later; see p. $16 f$. 
to be neither tall nor short nor of a medium height). Therefore as far as they belong to the subject, they are also referred to when we refer to it. We can try to present the scope of the reference in predication of both GS and GA in Diag. 2. Black spots represent objects we can refer to in an act of predication. Arrows connect inexistent attributes with their substance and the rule is that when we refer directly to an object we eo ipso refer indirectly to all objects connected with it by an arrow. In case of the direct reference to the very substance (e.g. man) there is the indirect reference to all contingent attributes of this substance (e.g. corpulence). But in case of the direct reference to corpulence there is no reference to other contingent attributes as they are not rooted one in another but all together in their substance.

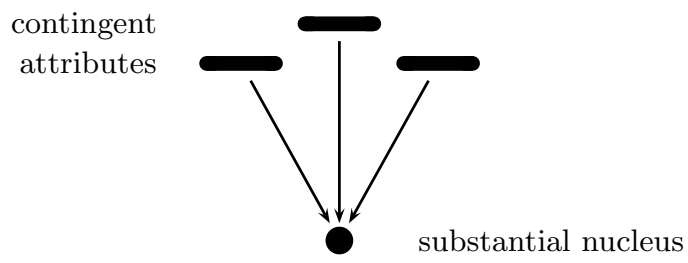

Diag. 2

There is another distinction among predicates in Aristotle. He claims that predication can be simpliciter (per se) or per accidens. ${ }^{4}$. Let us try to supply some explication of this dichotomy. Predication per accidens, literally: using an accident, is the case when some individual accident, part of the contingent equipment of a thing is focused on. It can be taken in a substantial context, e.g. when we say of somebody that he is a fatty. This case can be interpreted as a GS predication, because a fatty is by definition a tubby man. Evidently, it can occur without reference to GS, e.g. when we just say that Socrates is tubby. Predication per se takes place when no accidental requisite is relevant. Yet it does not have to refer directly to GS - it is sufficient to take some non-accidental, necessary attribute, e.g. rationality or mortality.

To make the difference between per se and per accidens predication more explicit we can draw content relations between predicates in Diag. 3, where rectangles represent predicates and thick lines mark species. A predicate is GS if it contains the category of substance (as e.g. animal, man, fatty), oth-

\footnotetext{
${ }^{4}$ See e.g. De Interpret. 21 a.
} 
erwise it is GA (e.g. rational, tubby). A per se predicate is always included in some species, i.e. it is always a (lowest) species, a genus or a differentia specifica (e.g. man, animal, rational). If a predicate is not included in any species it is per accidens. So eventually both divisions of predicates: GS/GA and per se/per accidens are built on the notion of species.

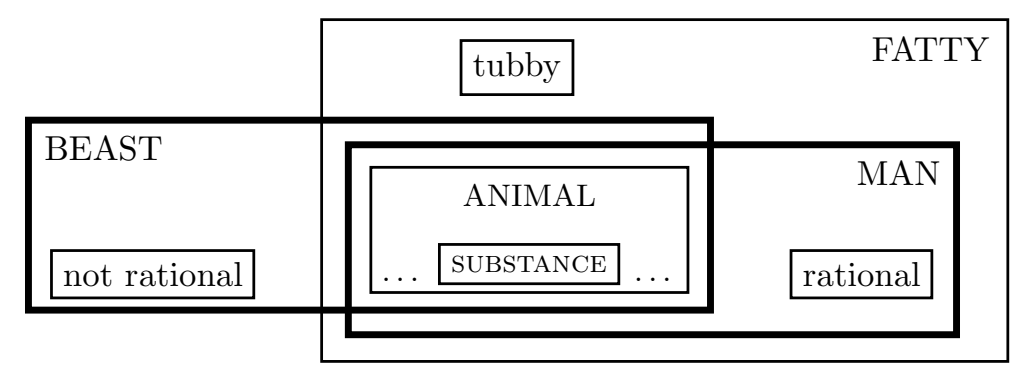

Diag. 3

In Diag. 3 predicates are related as if they were parts and wholes. Can we really apply these notions here? If we agree that predicates can be analyzed, divided and united, we come to the conclusion that there must be some reason for that. If they are complex beings they must have some parts. If they are not complex, what other principle can be given? It looks like in every possible explanation the notion of part is already involved. But a problem emerges whether such parts should be understood according to the content or the extension of predicates. In the first case rational is a part of man but in the second case the other way round. Is it not a matter of pure convention? The solution can be found when we look on the two extremes of the Porphyry's tree: a highest genus (primum genus) and a lowest species (ultima species). We would rather say that the first is simple and the other complex than vice versa. Of course it is not an conclusive argument but it surely supports the preference of content to extension here. The additional argument can come (for Aristotelians) from Philosopher's observations about some proportion among the relations between primary substances and their parts, on the one hand, and between species and genera, on the other (Cat. 2b 7-22).

Now, we can present the results of our investigations in a more detailed Diag. 4 (see the next page).

In each corner of the diagram there is a group of different entities (examples given beside). There are two kinds of relations holding between objects 


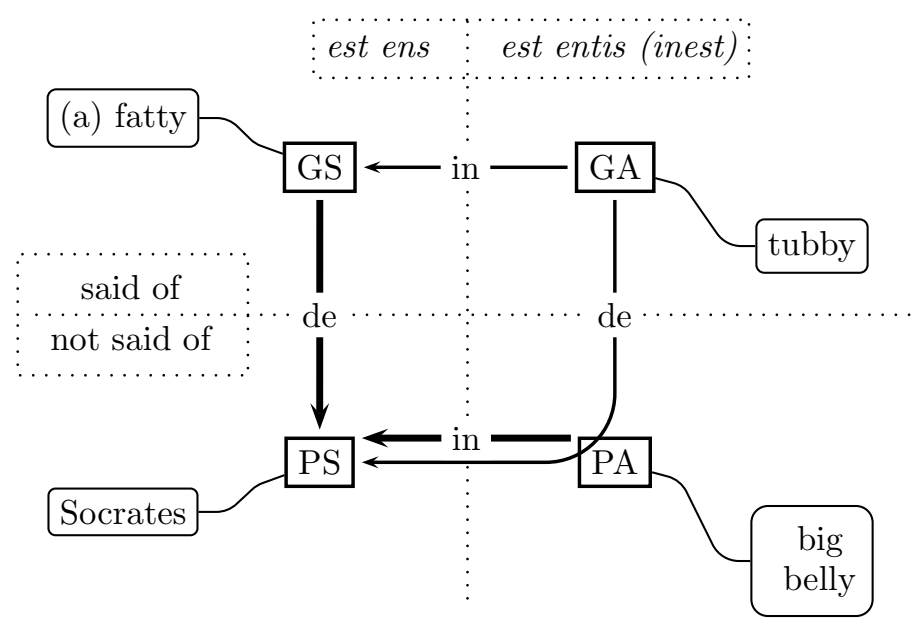

Diag. 4

from different groups: predicates are said of particular substances, attributes and parts belong to substances. ${ }^{5}$

\section{II}

In the framework presented in Diag. 4 certain reductions have been proposed. It seems that all these reductions were eventually intended to dispose of the relation of predication. They were originally presented in the context of the controversy about universals whence they got names of moderate realism, conceptualism and nominalism, respectively.

Of these three, conceptualism is perhaps the easiest to refute. The reason is its lack of determination, so to say. According to a standard formulation of this standpoint general predicates (universals) are not among things in themselves. All beings have to be concrete, particular. There would not have been universals without thinking (judging) subjects. Yet they are not on the side of linguistic signs. A conceptualist foresees devastating consequences of the nominalistic standpoint. So, he concludes, universals must be somewhere "between" reality and language — in the mind of the judging subject. The

\footnotetext{
${ }^{5}$ It cannot be ignored that some attributes are said not of the substances but of their attributes or parts. It is perfectly possible to predicate some property of an attribute or of a part of a substance: that crimson colour of Socrates' nose is intensive and his belly is big. Socrates himself is neither intensive (even with respect to colour) nor big. We will return to this subject later (see p. 20f.).
} 
mind has the ability of subsuming different things under one and the same concept. There is nothing common in things themselves - the only common thing about them is that they are thought about together. Common names result from this power of grasping things in classes: they are signs of our general concepts.

Let us try to follow consistently the line of thought of this approach. One should ask on what basis we collect different things under one concept. If the answer points towards any real entity independent of the mind we can call it conceptualism cum fundamento in re. The entity mentioned can be either general or particular. In the first case it would not be conceptualism but realism. In the second case we can ask further on: in what way does this particular serve as a basis for general concept? If it is a common part of all entities in question these entities are not separate beings - they are fragments of one being. This consequence is probably not more commonsensical as the realistic standpoint. But what is worse is that this principium generalitatis must remain purely hypothetical: it can be traced nowhere in the sphere of sensory perception. Then maybe all entities of the given class form the postulated particular as its distinct but complementary parts? This conjecture, known as the so called "theory of concrete universals", was actually made by some adherents of hegelianism. What profit may get a conceptualist here? If he claims that his mind can form a general concept of a triangle, either he must admit that he is capable of simultaneous presentation of all possible triangles or that he has not the concept of a triangle at all. Neither of these is true.

What seems to be left? Our factor of generality could stand in a particular relation to all members of the class or, at last, can be a particular relation itself. An object related to all objects subsumed under a general name could not be, by definition, one of these to which the general name refers. Nor can it be a specimen of the relevant attribute or part of such an object. In that case the bearer itself would be an argument of the relation instead of its attribute. The other possibility, however, seems promising. It is known under the name of "resemblance theory" since the relation connecting all members of a class is responsible for their resemblance. We actually recognize this resemblance and, the adherent of the theory claims that what we see is not the general feature identical in all members of a kind but different relative features of these members resulting from the same relation. The resemblance of a son $A$ to his father $B$ and resemblance of the father to his son is not $A$ and $B$ 's having some $X$ in common - it is $A$ 's having relation $R$ to $B$ and $B$ 's having relation $\breve{R}$ (converse of $R$ ) to $A$. Can we 
show how the general notion of a kind is formed on the basis of such a relation? It should be the notion: "being an argument of the relation $R$ ". What we have in reality, a conceptualist would say, are particular objects and their particular properties, among which properties like "being the $n^{\text {th }}$ argument of the relation $R$ " may occur but only for particular $n$ and $R$. The mental operation of generalizing consists in passing from such instances as "being $1^{\text {st }}$ argument of $R$ ", "being $2^{\text {nd }}$ argument of $R$ ", etc, to the notion "being $n^{\text {th }}$ argument of $R$, for some $n$ ". Properties in the point of departure are radically different: they are not specifications of the genus "being an argument of $R$ ". It is easy to see that not only conceptualism could profit from resemblance theory. It seems adoptable by nominalism as well. Actually, it deserves treatment independent of the traditional standpoints in the controversy about universals (see p. 10).

Coming back to specifically conceptualistic approaches we must consider what seems to be the last possible solution: the claim that nothing in reality is the foundation of the act of collecting things in classes; it is a completely spontaneous activity of the mind. We can call it conceptualism sine fundamento in re. Here we can also ask if it is the activity of an individual mind or the activity of every possible mind, of mind as such? In the latter case once again we have realism, more specifically its limited version claiming that general, i.e. identical in many different beings, is only certain mental structure. Nevertheless it is a realism. In the former case we deal with conceptual anarchism: "anything goes". The explanation of the fact that the species homo is constituted by all and only featherless bipeds rather than by some chairs, some tables and some of their users, is the matter of psychology, not of metaphysics. There is a controversy if it is a consistent standpoint but one thing can be settled for sure: the consequences are very serious, not to say devastating. Moreover, it seems that if such a radical step was made it would be natural to go still one step further and adhere rather to nominalism than to conceptualism. For, if the essence of the operation of generalizing is just collecting many items under one concept and then finding a name for this concept, simply inventing a name for a group of objects can be substituted for both steps at once. Berkeley in his Treatise drew such consequence of Locke's doctrine.

Thus nominalism seems to be a more radical but, at the same time, more consistently conceived standpoint. It can be expressed as follows: Every extralinguistic thing is individual. But there are certain linguistic tools which can be applied to talk of many different things at once: general names. Their generality consists in the semantical relation to their referents. This is the 
general framework of nominalism. The internal distinctions can be made according to the specification of the relation. First, we can ask if there is any objective basis for a group of things to be subsumed under a common name. Ex hypothesi it would have to be something individual. Let us search among the things in question and their parts. No identical common part of different things can be pointed out. Neither can it be simply everything that there is in these things as well - apparently some of their aspects are irrelevant from a general point of view, e.g. no change of colour of a pair of shoes can stop them being shoes.

At this point the conclusion is that in every object belonging to a class there must be some different part responsible for its belonging to this class. These parts constitute a class again and the question of its objective basis can be raised anew. Are we starting a regressus in infinitum in this way? At least in some cases it can lead to an endless process but in our opinion it would be a progress rather than regress. The situation can be recapitulated as follows: For every set of objects falling under one general name there is some basis of this correlation: a set of certain parts of these objects. If we are able to give particular grounds for every case of a general name it is all we need. If such basis, being a set, can be again referred to by means of a general name, still another basis is demanded. Nevertheless, this second basis is not a conditio sine qua non of the first. It is needed only in case of another general name. However, an objection can appear about the ontological status of a set. In the nominalistic framework, one can say, only individuals are beings and if we look for an ontological basis for some general name it cannot be anything but a being, one being. Now, if somebody indicates a set of beings it must be proved that it is not simple plurality but one being. And thus the initial problem of the basis of this unity recurs. This time a regressus seems inevitable. Cannot really an ontological basis for a general name be plura entia instead of unum ens? Let us try to call the whole thing otherwise. It is not the single basis for a general name that is necessary, it suffices to have many bases, one for each separate object of the reference of the name.

Let us look closer at these separate bases now. Each of them is a certain part or attribute of the relevant object. As a result each object has some part or property which absolutely cannot change if that object is to preserve its general name. We have already mentioned that the colour of a pair of shoes is not essential for shoes in general. What is essential then? To our surprise we must acknowledge that the border between essential and accidential properties cannot be found among individual parts or properties: each of them can change (to some extent) without harm for the general characteristics of 
its bearer. We cannot catch the species on the surface of the individual so to say. We must reject the first account for the generality of names then: its principle cannot be a particular set of parts or attributes of the relevant group of objects. If we still insist on such an objective principle we must look somewhere outside the objects collected under a general name. Remembering that no ideal type is allowed for an honest nominalist, we come once again to some individual relation between our objects. It is the resemblance theory again. Before further treatment of this standpoint let us remind the most radical solution: names are attached to their objects of reference entirely arbitrarily or at the utmost on the grounds of a linguistic convention. The result of this approach has been already mentioned (see p. 2).

Now we may set to the investigation of the resemblance theory. In our opinion if it proved to be consistent and able to give a nontrivial explanation of general names it could serve as the only justification for nominalism. For the sake of simplicity let us assume for the time being that all referrents of a general name are given simultaneously. A particular relation of resemblance holds between all of them and only them. This relation, as the adherents of the theory claim, serves to distinguish the set of the objects of reference of the relevant general name. Each such object, as an argument of the mentioned resemblance relation (let us call it $R$ ) is a bearer of a relational property "to be $n^{\text {th }}$ argument of $R$ " (for a stated $n$ ). Does not this relational property falsify our earlier statement that no individual property of an object is relevant to its general characteristics? When we look at things closer, we can say that it does not have to be the same relational property all the time. In the process of change the arguments of the relation remain similar. But it does not mean that the same relation $R$ holds between them, of course. It must be a new relation $R^{\prime}$ after a change in the objects. And accordingly the objects lose relational properties based on $R$ and gain analogical properties based on $R^{\prime}$. But if so, what guarantees that $R$ and $R^{\prime}$ constitute the basis for the identical general name? A relation of higher order between these two resemblances? Such a super-relation would have one nonexistent argument, namely $\mathrm{R}$ which ceases to hold just when $R^{\prime}$ starts.

Is such a relation compatible only with a general acceptance of individuals? Each individual object exists only if all its parts exist but can a relation be treated in the same way as a nonrelational object? Not quite! E.g. individual nonrelational objects exist in space but individual relations between them do not exist in space between them. Could it be by analogy maintained that relations between objects existing in time do not exist in time? Such a supposition would lead to a conclusion that attributes do not 
exist in time either. ${ }^{6}$ It would be as if subjects bore certain relations and properties at particular time and place but relations and properties were somehow beyond time and space. There is no sense in asking what exactly happens before certain existing objects enter such an atemporal relation. It is true that if the relation is not necessary, the sole existence of objects is not enough for it to hold. One can ask then, what is the sufficient condition for it to start holding. Neither new objects nor any other relation (regressus!). No attributes of the objects could help (they are also atemporal). The objects just enter the relation and this is all. This standpoint would help us much because the continuous existence ceased to be a necessary condition of identity for attributes and relations.

However, even after removing relations from temporal region the problem of their nonexisting arguments remain. Cannot there be a similarity between, say, me and my great-grandfather? Moreover, the relation of temporal precedence has arguments which do not coexist. So it seems that we have grounds for accepting relations between arguments existing in different times. When does such a relation hold? Not before any of its arguments. The necessary condition for it to hold is that all its arguments have already come to existence (but not necessarily continue to exist). We agree on relations with already nonexisting arguments but not on relations with arguments which have not yet existed. To avoid misleading suggestions we should rather say that arguments enter a relation instead of saying that the relation starts (and stops) holding between them. If relations are not spatio-temporal entities then our original trouble disappears: The two resemblances connected by the super-relation neither exist nor do not exist. Their holding or not is something about their arguments rather than about themselves. They do not have to hold then, to be related to each other.

But we have to remember that the present ontological framework admits no general entities. And atemporal relations resemble ideal beings. Therefore the ideal (atemporal) must be distinguished from general. Protagonists of the resemblance theory who reject not only general beings but atemporal and non-spatial individuals as well, must accept certain web of relations between relations. Let us have a closer look at this alternative. Suppose that from time to time a new individual appears in a given kind. Each time there starts a new relation of resemblance: $R, R^{\prime}, R^{\prime \prime}, \ldots$ There must be some super-relation $S$ which occurs between $R$ and $R^{\prime}$ at the same time as

\footnotetext{
${ }^{6}$ This opinion is not as bizarre as it seems at first and was given support from authorities - see e.g. R. Grossmann, The Existence ..., p. 5.
} 
$R^{\prime}$ occurs. $S$ is necessary to secure that $R^{\prime}$ is "similar" resemblance as $R$. But this $S$ cannot reach $R^{\prime \prime}$ before the latter starts. So in proper time some $S^{\prime}$ between $R, R^{\prime}$ and $R^{\prime \prime}$ must occur and consequently some relation $T$ of a still higher level between $S$ and $S^{\prime}$ as well. With each new object not only a new resemblance but a new higher level of super-relations is neessary as well (see Diag. 5).

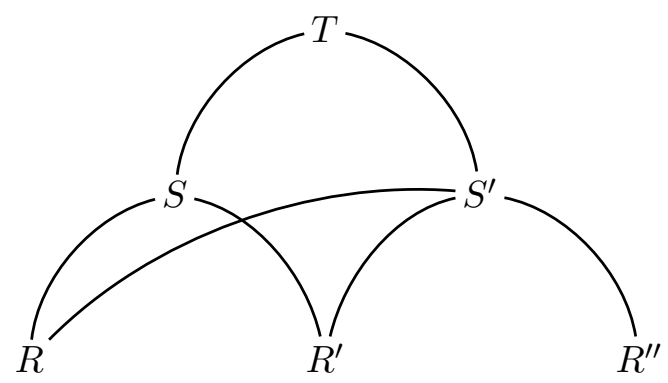

Diag. 5

Newer and newer super-relations serve to collect and unify older relations into one system. Without that the temporal identity of species would be lost. Is this constantly growing web of relations a component of the species itself? In such a case the species would change with the appearance of each new individual. However, species are not individuals which can subsist through changes. Relations constitute species but are not its components. But the sole arguments of $R, R^{\prime}$, etc. cannot constitute species either. So the latter option concerning the nature of relation must be rejected if we are not to lose the notion of the immutable species at all: relations have to be atemporal for the sake of species immutability.

Let us now try to define once again what the species is in this perspective. When we say that Socrates is a man we assert that he resembles certain other individuals (both contemporary to him and already passed away) and that this factual resemblance is related to certain other resemblances some of them have already ceased to hold and some have not yet started holding. Hence the object of our predication here is some complex individual relational property. It is individual because we must remember that we are not asserting being an argument of a given relation in general, but being a particular argument. And for every object the above property is different. In time the object changes its place in the web of relations - it passes from the 
resemblance $R$ to the resemblance $R^{\prime}$ and so forth (see Diag. 6). However, in this passing through resemblances the web itself remains invariable (as an atemporal complex individual):

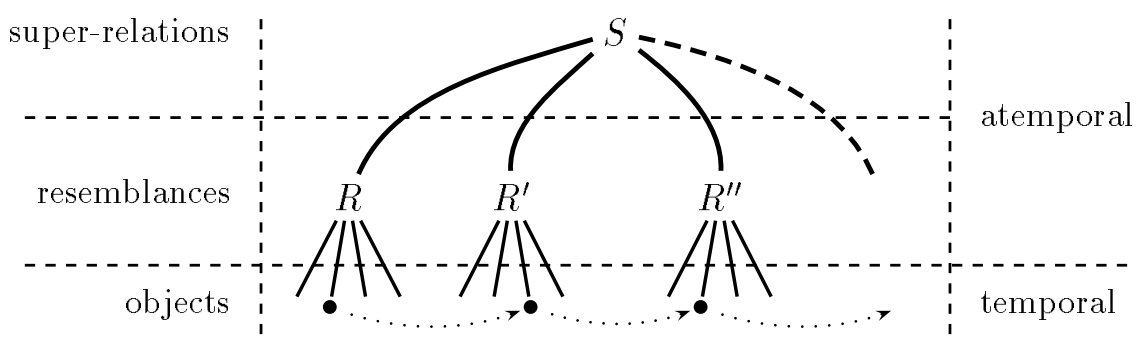

Diag. 6

It is easy to notice another peculiar feature of the resemblance theory. Relational properties described above cannot belong to an individual unless there is (or was) at least one individual similar to it. Generality is thus interpreted in this theory as factual plurality. Could it really be maintained that Socrates is a man only if at least Xantippa is? Another problem is whether the resemblance theory enables us to distinguish between GS and GA. We have characterized this difference between predicates using the categorial notion of substance (see Diag. 3). How would the resemblance between attributes differ from the resemblance between substances? Let us compare two predicates like "a fatty" (GS) and "tubby" (GA) in Diag. 4. In a realistic interpretation the difference is just presence of the predicate "substance" in the former one. Can that have an equivalent in the framework of the resemblance theory? Surely there cannot be any resemblance between pure substances, so maybe a difference between resemblance of individual substances on the one hand and resemblance of individual attributes on the other can be discovered? But if attributes are arguments of the resemblance relation the corresponding relative property is a property of an attribute, not of a substance. If this shade of red resembles that one, the similarity to a given shade of red is a characteristic of the other shade of red only and not of its bearer. Realistic premisses have allowed us to find the difference within the internal structure of predicates, which in turn enabled two kinds of reference: to the object as such and to the object with respect of some its property. Could we reconstruct that as the resemblance of objects as such and the resemblance of objects with respect to certain attributes? It is nec- 
essary to remember that now we no longer have the difference between PA and GA. So PA cannot serve as a respect of predication of GA. All attributes are $\mathrm{PA}$ and when we have predication of a relational $\mathrm{PA}_{1}$ no other attribute $\mathrm{PA}_{2}$ can serve as the specific respect of this predication just because the second is quite another particular attribute. There is nothing except individual objects and their individual attributes. What is said of an object is always some (relational) attribute and there is nothing more in the object to serve as a respect of this predication. So the result is that within the present framework predication has homogeneous character. It is always predication of attributes. Predication of a substance is impossible because this would imply that peculiar character of a substance, independent existence, could be something relational, which is contradiction in terms. What an object in its very depth is - a self-sufficient being - cannot rely on anything else. It is rooted in the object itself and would not disappear even if there was nothing else around.

What conceptualism, nominalism and resemblance theory have in common is their rejection of any objective general entities. In our Diag. 4 it must result in substituting something for both GS and GA. As we have seen both conceptualism and nominalism can be cum fundamento in rebus or sine fundamento (arbitrary). The first case can be explained in terms of the just discussed resemblance theory. In Diag. 4 certain complex individual properties substitute GS and GA then. In the other case no specific individual entity is at hand. To say that Socrates is a man is just to say that he has been included into certain group of objects joined by some mental operation or a common name. In other words, Socrates was thought of as a member of some group or that he was given a name "man" together with other objects. When predication is grounded in individuals in our Diag. 4 both GA and GS change into PA. As PA is not general it is no longer a predicate and the relation of predication disappears. Now we just have a part-whole relation. Obviously we can still speak of predication in the sense of an mental or linguistic act, but there is no longer predication in the sense of an objective relation. Moreover, from the point of view of fundamentum praedicationis there is no difference between predication in the category of substance and predication in the category of attribute. Perhaps this consequence partially helped modern empiricists to criticize at first and eventually reject the notion of substance. When it comes to sine fundamento in rebus versions of conceptualism and nominalism, just nothing objective is substituted for (general) predicates. It cannot be said that some sets of individuals are substituted there. Sets in the distributive sense are not to be 
found in the world of individuals. Mereological collectives contain far more ingredients than only relevant objects of predication and if we tried interpreting predication as the belonging of an element to such a set we would be obliged to accept that not only Socrates is a man but his nose as well. So in these variants of conceptualism and nominalism the upper level of Diag. 4 is simply suppressed.

Now the time has come to investigate the last and least radical of reductionistic tendencies: moderate realism. It is ready to accept the general beings but only on condition that they are somehow connected with individuals and do not exist separately from them. This connexion is better understandable in the context of Aristotle's system. He regarded predicates as forms of individuals. Forms understood like shapes of bodies cannot exist apart from formed material. But an interpretation like that is likely to obliterate the difference between a universal and an individual attribute: a shape is something that a thing has, not something that it is and secondly, it is something individual - each thing, no matter how much similar to another, has its own peculiar shape. This shape cannot be reproduced anywhere else. So the interpretation of Aristotelian doctrine of universals as forms has to avoid their "particularisation" ${ }^{7}$ Forms particularized cannot be universals: general beings predicated of individuals. Why did Aristotle insist on the immanence of forms? Paradoxically, perhaps he wanted to avoid making forms and individuals too similar. For one thing, independent existence was for him the very fundamental feature of a particular being. (In Plato, form used to be interpreted as a model, distinguished specimen of subordinate individuals. Thus it seemed to be an individual par excellence.) And secondly, two beings existing independently would not compose one whole without some ontological glue. One of them must have the nature of an absorbent and the other has to be assimilable by nature. Now the absorbent is the form, and the matter is that component of an individual thing that is absorbed by the form. In this process of absorbing neither the form splits in parts corresponding to separate portions of matter nor these separate portions of matter merge into one big piece; general (universal) character of the form consists just in that. And form is inexhaustible: it can absorb any number of individual portions of matter, they cannot clog it. In our opinion the doctrine of the immanence of forms stresses their "absorbing" nature. They cannot exist as only potential absorbents, they have to absorb actually. (The real

\footnotetext{
${ }^{7}$ In spite of the support given to this tendency by such prominent authors as Reale in his history of ancient philosophy or Anscombe in Aristotle.
} 
absorbent does not have to absorb liquids all the time, but this is because each thing is always partly actual and partly potential). If the form is that factor which brings in actu esse to a thing, this factor itself cannot exist as something potential. Aristotle's statement: "actus est prior potentia", i.e. "ex potestate ente fit actu ens ab actu ente" 8 could be perhaps interpreted as the condition that a form can exert its absorbent action on matter only as embodied in some matter already. So matter would serve form as a kind of starter. An individual thing has its matter and the form and accordingly to that moderate realists express their thesis saying that universalia (i.e. forms) sunt in rebus.

Let us now try to find a satisfying interpretation for this thesis which is very relevant to our main topic. Interpreted verbally, it would lead to the same suppression of the upper level of Diag. 4 as before. But we should distinguish two things now: Does esse in the thesis above mean existence (as if universalia-in-rebus or -cum-rebus sunt) or the part-whole relation (universalia sunt-in rebus, universalia sunt rerum)? Only the first rendering is plausible in our opinion. This means that existence is not something that could be said of a pure form. It is only the embodied form which exists or, better, we can talk of the existence of forms only in the context of existing things. It is a particular thing which exists in a proper sense while form exists only derivatively as something which constitutes the existing thing. Instead of universalia in rebus it would be more appropriate to say universalia per res. Existence has temporal character and universals in themselves, as atemporal beings, are beyond this qualification. Only their instantiation in individual things can exist. However, even when no instantiation takes place, universals are something, are beings. Avicennian doctrine of natura tertia could be of some help here. The instantiation of species in individuals is one thing but species in itself, apart from both its general character and its instantiations is still another. It is neither general nor individual ("equinitas est equinitas tantum"). ${ }^{9}$ Dependence of existence is not dependence of being. ${ }^{10}$ As beings they are something in themselves and what they are neither depends on anything nor is related to anything else. This can be said to

\footnotetext{
${ }^{8}$ See Met. $\Theta 1049$ b 5, 25.

${ }^{9}$ By the way, this neutral quidditas tantum was used to try to explain the nature and possibility of the very relation of predication. As if its status prior to both generality and individuality could serve to link both. But it is easy to see that such an approach only shifts the problem to a higher level.

${ }^{10}$ This difference is rendered very clearly in Ingarden's distinction between existential moments of dependence and heterogeneity.
} 
be the general thesis of realism. Thanks to that, species, even if not instantiated, does not lose its being, quidditas. Being a man is something even if no man exists. There is no horror vacui formarum then: continuous existence of individuals is not necessary to secure identity of species. Summarizing, we can say that the moderate version of realism accepts general beings but rejects their independent existence. In this light the doctrine does not lead to any reduction in Diag. 4 but, on the contrary, places the two levels of the diagram, individual and general, in a proper distance. Thanks to that the relation of predication can hold: predicates are in need to be instantiated and individuals are their subjects.

\section{III}

Nevertheless reaching the realistic standpoint in the controversy about universals is not a final step. All of the aforementioned would-be reductions were aimed at suppressing predicates and their relations to individuals. If we had been lucky to carry out any of them, we would not have had to explain what a predicate and its relation to an individual really are. So now we must at least admit that this task is still before us. By no means it is a minor objective: its performance, comprising a detailed and satisfying description of the items in question, could be the eventual resolution of the controversy about universals. If our investigations presented here have been conducted properly, we proved no more than that the problem of universals is still looking for a resolution. But having confessed that we are unable to cancel the problem of universals we can still try to limit its extension so to say. It seems that the region where universals can be disposed of is GA predication. Predication of this sort serves to qualify things. It seems plausible that a description of what a thing is like involves some relations to other things. In other words, without relation of comparison things would be no more such and such. The opinion that a thing is qualified only relatively to others has quite a long tradition in philosophy. Something at last similar is expressed already in Plato's Theatetus 202 D, and the well known argumentation against the reality of the phenomenal world in Bradley's Appearance and Reality is based on this assumption. In any case we see no counterintuitive consequence of assuming that all qualities are of relative nature. However, this is not the case with GS predication, as we have already observed (see p. 2). It seems reasonable then to assume realistic position (in moderate version) with regard to GS predicates and to retreat on resemblance theory 
ground with regard to GA. The profit consists in substituting atemporal but individual beings (relations) to atemporal general beings (predicates).

But, one might say, is such an atemporal and non-spatial being really individual? Some people tend to regard this characteristics as a mark of an ideal being and what is ideal is often identified with general. ${ }^{11}$ In our opinion the most fundamental character of a general being is a feature which is sometimes called incompleteness. Lockean "triangle in general" is neither right-angled nor acute nor obtuse. Because of that the rule of excluded middle does not apply to universals. This holds even in the case of the lowest species. There is a sharp difference with respect to that between predicates and dependent parts of individuals like, e.g. this particular red colour of the beret of my girlfriend. A particular attribute is something individual and even in itself, i.e. in abstraction from its substratum retains full completeness. This red shade has been abstracted as something particular, mentally cut off. But species "red" cannot be abstracted in the same way. Husserl calls to the process of passing from an individual object to its species an abstraction too, but the GENERALIZING abstraction. It does not consist in separating some group of particular attributes from the rest of particular attributes but it demands leaving the level of particularity altogether. If we pass from Socrates to a featherless biped we ignore not only the hair of the animal in question - we have to ignore all particular characteristics of its legs as well (their duality is nothing particular of course, it is duality in general). And when we pass from a species to a genus there is already nothing particular in the first to be omitted: the move consists in ignoring some already GENERAL characteristics (e.g. double number of legs, lack of feathers, etc.). We do not omit any particular pair of legs nor do we ignore the lack of any particular feathers. So the incompleteness of a predicate should not be understood as some kind of an ontological "hole" in a real thing, it rather spreads over the whole (general) object. Speaking exactly, what we have here is not incompleteness (where some remainder of a complete particular is assumed): it is some altogether different kind of being. In other words, there can be no ontological mixture made of particularity and generality: these two spheres are always separate. Atemporality and non-spatiality of general being is the consequence of this radical separation: otherwise sepa-

\footnotetext{
${ }^{11}$ In Ingarden we find a reign of so called pure ideal qualities which have simultaneously ideal and individual character. But this thinker makes a difference between such a quality and its so called concretization. With regard to atemporal relations it would probably lead to a distinction between relation as such and relation holding on its arguments - a complication which we would like possibly to avoid.
} 
ration could not have been secured. The only relation which holds between general and particular is the relation of predication. ${ }^{12}$ From the presented point of view one can see more clearly that a resemblance relation between certain specified particular elements cannot be a general being: it has to be as particular as its arguments although it is not situated in time and space limits.

Can the difference between GA and GS interpreted with the help of resemblance somehow correspond to an Aristotelian statement that there is no science of accidents? If a specified object (resp. field of objects) is a necessary condition of each science there cannot be a science investigating the nature of the resemblance relation. The relation itself is primitive and the only thing which can be said about it is that it holds between such and such objects. But this says nothing about the relation itself. Still another thing is that Aristotle himself and after him all mainstream logicians up to Frege and Russell used to disregard relations. Their ontological status seemed doubtful ("There is no attribute having one leg here and the other there" as Leibniz used to say). For Aristotle a relation to a being is a being too (in the analogical sense) but perhaps it is not "dense" enough to become an object of scientific investigation.

Let us finally consider some non-traditional conception of universals which could supply some indication as to the understanding of universals. It is a standard argument in every refutation of nominalism that this standpoint renders any general laws, and hence any nomological science, impossible. But maybe this is more than a straightforward consequence of nominalism. It could be just its reverse side. Namely, realism could be understood as a standpoint which, instead of asserting existence of general beings, asserts presence of general laws. What is the difference? In this perspective there would be no general beings, predicates would be just linguistic tools serving only to express the law, and the essence of the law would consist in subordinating certain number of individual beings to some general rule. Predicates could be understood as variables having a definite range of variability. The correspondence between a general name like mass, force, etc. and physical objects would consist in the applicability of a law in which the given name occurs to such and such individuals. Laws could be understood analogically to axioms of geometry implicitely defining elementary terms like

\footnotetext{
${ }^{12}$ For moderate realists this tie is too weak (cf. Aristotelian objection of $\left.\chi \omega p \iota \sigma u o ́ s\right)$. Hence they can try to "stick" the two spheres together with the help from the part-whole relation. But the platonic (extreme) realists have to explain how incompleteness of the general makes predication possible.
} 
point, straight line, etc. by prescribing certain rules for them. It is not the nature of the object to determine laws of its behaviour but vice versa: the nature (specific essence) of the object is determined by rules and eventually the very nature of the object is nothing more than rules of its behaviour. The proper species of the object is determined by its subordination to such and such rules. Objects obeying the laws could correspond to a range of a variable represented by a general name occuring in the context of the law in question. From a syntactical point of view the difference between a traditional version of realism and the present approach boils down to a difference between category of name and category of sentence. The ontological "source" of generality would be not predicates but propositions expressing laws. Is not such an approach intuitive? When we want to classify a given object as a e.g. piece of gold do we look for any particular attribute of the thing or rather we check if it fulfils certain tests? In the second case we look for something which was often called power, faculty or primary quality (as opposed to secondary one). What we check in this case is the subordination to a general rule. When we check somebody's rationality we do not look for any particular thoughts but for the ability of thinking (we try to stimulate certain mental processes in a stated way). But do we always look for faculties instead of actual properties when we classify things? We can remind here a distinction made by Locke and his inheritors: primary qualities were identified with essential properties and secondary with accidental ones. Can we say that one looks for properties of this first kind in determining the kind of a given thing and that properties of the latter kind are looked for when one asks what the thing is like? It would be an oversimplification. Nevertheless, we can divide actual properties of a thing in two: in the first class there will be properties whose presence can be subsumed under some general rule (they can be conceived of as values of some function). In the other class there remain all properties which cannot be treated in this way. Now, all faculties of a thing and their manifestations can be treated as composing the species of that thing. All remaining properties could be counted as accidental and only a resemblance could hold between them.

As a supplement let us look closer at the problem of predication of attributes of other attributes. The situation presented in Diag. 4 iterates: GA is predicated of some $\mathrm{PA}_{1}$ with regard to a $\mathrm{PA}_{2}$ of this $\mathrm{PA}_{1} \cdot \mathrm{PA}_{2}$ of $\mathrm{PA}_{1}$ is, in our opinion, a specific instance of the problem of so called intermediate parts of the whole (Twardowski, Husserl et al.). As we have remarked earlier, in Brentanian tradition attributes were regarded as a special kind of parts whose existence depended on existence of other parts of the rele- 
vant wholes. Because of this feature they were called dependent parts. Now, example given in the footnote 5 clearly shows that in some substances we can find attributes which are not attributes of the substances directly but rather attributes of some parts of these substances. As Twardowski remarks, they can be regarded as parts of the relevant substance in a derivative sense only. ${ }^{13}$ Their immediate substratum is always some part of the substance so at first they constitute a whole with it, and only in the context of such a whole they belong to a substance. They are parts of this substance only via other parts. When such a go-between part is itself an attribute we have the situation in question. Now the following question can be raised: Can only PA be a subject of predication or GA as well? The last case is similar to the earlier problem of the nature of the relation between GS and GA. We have argued that it is not predication but part-whole and now the argument has to be similar. For the first thing, there is no PA contained in GA which could serve as a regard of the predication. Besides, if we say that, e.g., rational $\left(\mathrm{GA}_{1}\right)$ is alive $\left(\mathrm{GA}_{2}\right)$ it is evident that the subject of the predication is not $\mathrm{GA}_{1}$ (no GA can be alive) but some PS serves as a subject for both $\mathrm{GA}_{1}$ and $\mathrm{GA}_{2}$. It seems that when we find an example in which an attribute would not be said of an PS but of $\mathrm{GA}_{1}$ only (red is intensive, e.g.), the subject of the predication would not be a general but particular attribute. But what about the predication: white $\left(\mathrm{GA}_{1}\right)$ is neutral $\left(\mathrm{GA}_{2}\right)$ ? White is here the only subject of the predication. No white thing is neutral in this sense. After a moment of reflection we must say that here again the true subject of the predication is particular, only the proposition is quantified: White is (in every case) neutral. It is obvious that only particular instances of white have the neutral character: white in general cannot be seen so it cannot be neutral either.

The next question connected with the problem of the attribute as a subject of predication is the following: is such a predication always made with regard to some attribute $\mathrm{PA}_{2}$ of an attribute $\mathrm{PA}_{1}$ or can it be made straightforward? E.g. can we say not only about this piece of chalk that it is white but also about its white colour itself that it is white as well? At first we see no objection against such "reflexivity". It seems that if white had not been white itself, white things could have not been white. However, it cannot be always the case. When PS is Socrates, PA - his age and GA - old, we can say of Socrates with regard to his age that he is old but we cannot say about his age that it is old, too. Of course, the expression "old age"

${ }^{13}$ See Twardowski, p. 47. 
exists but cannot be understood literally: "to be old" means something like "to have relatively long history" and surely nobody's age can have a history; only a substance can have it. Similarly with Socrates' weight and predicate "heavy". The reason of this nonreflexivity is probably the following: When what is being predicated consists in having some specific part (e.g. two legs in the case of the predicate "biped"), PA being "the regard" of the predication is a property of the RELATIONAL nature. The relation holds between the subject of the predication and something different. If the same predicate has had been applied to this relational property itself, the relation would have had to hold between the same property taken twice. White is not a relational property and that is why white being white does not imply the paradox of a relation having one argument only. So eventually, when we take into account predication about attributes, Diag. 4 can be extended as follows:

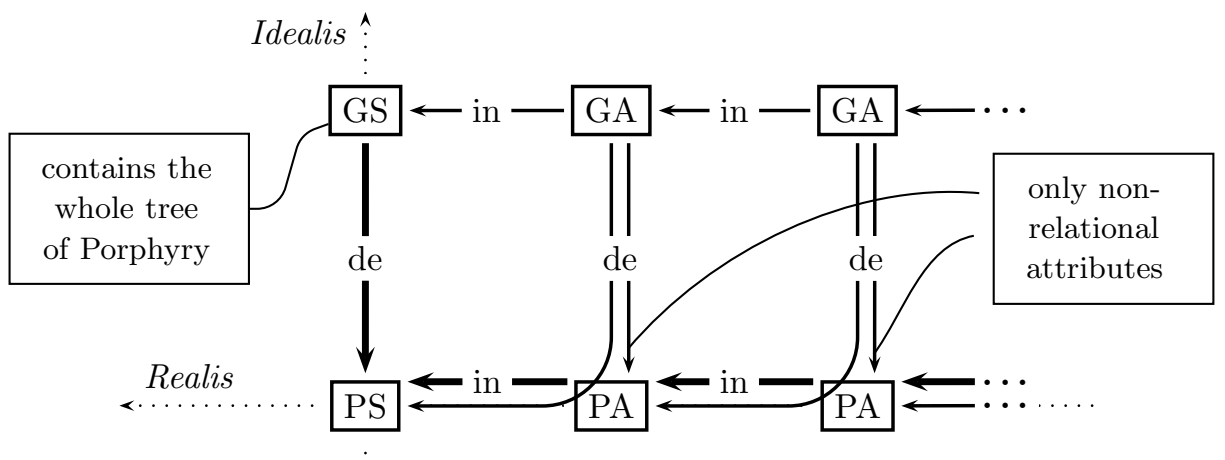

Diag. 7

The order of predicates in the upper row seems to repeat the order of particulars below. But it is not exactly so. While (according to Aristotle) no PS is a part of another PS, some GS (genera) are parts of other GS (species or lower genera). The order of genera contained one in another is Porphyry's tree (not represented in Diag. 7, for the sake of simplicity) and it has no counterpart on the side of particulars. Seen in this light, one of Aristotle's objections against the realistic standpoint: that universals just duplicate the world of particulars, cannot hold.

What general picture does our final diagram show? The entities are displayed in an order reminding a coordinate plane. The two dimensions of the 
plane are constituted by the part-whole relation and the predication relation, respectively. We could call them realis and idealis. In this system of coordinates PS is the centre. Subsequent parts of parts, attributes of attributes have less and less reality with regard to PS. But nowhere in this dimension the ideal can be found because ideal is not a part of real. Passing from the real to the ideal needs entering a new, independent dimension. The peculiarity of this dimension lies in its binary character: 0 is real, 1 is ideal. Higher genera are not more ideal than lowest species: their hierarchy only repeats the part-whole order on an ideal level and thus is displayed horizontally, not vertically. None of them is predicated of another: all are predicated of PS.

The main result of these investigations can be summarized as follows: The order of beings cannot be reduced to one dimension only. The two dimensions are mutually independent, which means that the part-whole relation can appear on the level of predicates and predication can be made about pars of any level.

\title{
References
}

Anscombe, G. E. M., "Aristotle" [in:] Three Philosophers, Basil Blackwell, 1961.

Armstrong, D. M., Universals and Scientific Realism, vol. I-II, Cambridge University Press, 1978.

Grossmann, R., Ontological Reduction, Indiana University Press, 1973.

Grossmann, R., The Existence of the World, Basil Blackwell, 1992.

Ingarden, R., Spór o istnienie świata, III ed., vol. I-III, PWN, 1987.

Martin, G., General Metaphysics. Its Problems and Methods, transl. D. O'Connor, George Allen \& Unwin, 1968.

Reale, G., Storia della filosofia antica II, IX ed., Pubblicazioni della Universitá Cattolica Milano, 1992.

Twardowski, K., On the Content and Object of the Presentations, transl. R. Grossmann, Martinus Nijhoff, 1977.

\author{
MAREK RosiaK \\ Department of Logic \\ Lódź University \\ ul. Matejki 34a \\ 90-237 Lódź, Poland \\ e-mail: rosiak@krysia.uni.lodz.pl
}

